\title{
Theory of Dielectric Relaxation for the Three-Dimensional Polar Rotator: Lattice Models Leading to Bimodal Loss Curves
}

\author{
John D. Hoffman and Benjamin M. Axilrod
}

\begin{abstract}
The characteristics of the dielectric relaxation spectrum associated with some simple three-dimensional lattices consisting of polar molecules of specified shape have been investigated with the object of determining whether dielectric loss curves with two distinct maxima (bimodal loss curves) could be predicted on a theoretical basis for such systems. For the types of lattice and molecular shape considered, each dipole has one stable and four metastable orientations.

The calculations show that under certain circumstances bimodal loss curves may arise for pear-shaped molecules in both the body-centered orthorhombic and tetragonal systems. All of the models lead to a single loss peak if the barrier system is sufficiently isotropic as in the body-centered cubic lattice. The changes with temperature of the shape of the loss curves, the static dielectric constant, and the configurational entropy are discussed. The abrupt changes in these properties that will occur at phase transitions due to cooperative interaction are also considered. Qualitative predictions concerning the expected behavior of bimodal loss curves for three-dimensional lattices, in which each dipole has only a single stable orientation, are compared with experiment.

Using an argument based on the fact that broadened loss curves in monophase molecular crystals become narrower with increasing temperature, it is concluded that fluctuations of structure are not the principal cause of the broadening of loss curves. The present theory leads to the correct type of temperature dependence, and this strengthens the view that the origin of multiple relaxation times in molecular crystals is the anisotropy of the crystalline field.
\end{abstract}

\section{Introduction}

It is well known that for many molecular crystals in which the polarization decay mechanism is the result of the reorientation of permanent electric dipoles the dielectric relaxation spectrum is best repre. sented by a set (or distribution) of relaxation times rather than by a single time constant. This is indicated by the fact that when the experimental values of the dielectric loss factor for such a material are plotted in the usual manner as a function of the logarithm of the measuring frequency, a bell-shaped loss curve is usually obtained which is considerably broader than that predicted on the basis of a single dielectric relaxation time. In some instances, a plot with two distinct maxima (bimodal loss curve) is found.

One of the main objectives of this paper is to show how an extremely wide set of relaxation times capable of leading to a bimodal loss curve can be predicted theoretically for systems that, for reasons of lattice symmetry and molecular shape, may reasonably be assumed to consist of three-dimensional polar rotators with five orthogonal orientations. Hitherto, calculations on the three-dimensional polar rotator have not been closely related to details of molecular shape and lattice structure, and have yielded only a rather narrowly spaced set of relaxation times leading to a somewhat broadened loss curve [1], ${ }^{1}$ or a very general type of information not concerned with the details of the shape of the loss regions [2]. Bimodal loss curves have been predicted for one special type of single-axis rotator [3].

\footnotetext{
${ }_{1}$ Figures in brackets indicate the literature references at the end of this paper.
}

Another objective is to deduce from the theory the general characteristics to be expected of bimodal loss curves in molecular crystals. These qualitative predictions, which are not in general sensitive to a special choice of models, are compared with experiment.

A quantitative comparison of theory and experiment is considered to be unwarranted at the present time. In the first place, for reasons of simplicity of treatment, we are forced to deal with the bodycentered orthorhombic (bco) and body-centered tetragonal (bct) systems, whereas the best dielectric relaxation data now available for three-dimensional polar rotators are for the face-centered orthorhombic $(f c o)$ and face-centered tetragonal $(f c t)$ forms of the hydrogen halides. Nevertheless, with certain approximations to be mentioned, the results for the $f c o$ and $f c t$ lattices are practically identical with those obtained for the $b c o$ and $b c t$ types. The second and more serious complication is that the question of the exact position of the hydrogen atoms in the hydrogen halide structure is unsettled, with the result that even the most stable dipole orientation is uncertain.

Several specific three-dimensional models are discussed. All are based on pear-shaped polar molecules situated in a $b c o$ or bct lattice. The time-dependent dielectric properties of each of these models are illustrated by predicting the shape of the loss curves for certain specified arrangements of the activation energy barriers hindering reorientations between the various possible positions (sites) that each dipole may occupy by rotating about its lattice point. In addition, an equilibrium dielectric property, namely, the total orientational polarizability, is 
calculated for each of the models. This provides information concerning the behavior of the static dielectric constant. Consideration is also given to the effect of change of temperature on the shape of the loss regions, the static dielectric constant, and the configurational entropy.

The methods used to calculate the molecular relaxation times and the orientational polarizability associated with each relaxation time have been discussed in considerable detail for three-dimensional lattice models in an earlier publication [1]. ${ }^{2}$ Therefore, after describing each model and setting down the differential equations pertaining to it, the relaxation times and polarizabilities are cited without giving the details of the calculations. The loss curves were calculated from these quantities with the help of eq $(2 \mathrm{~b})$ of the following section.

The results obtained in reference [2] regarding the general properties of the relaxation times were used to check the results quoted in this paper. In addition, the general formulas valid for orthogonal sixsite models given in the appendix of reference [2] were used to check the calculation of the total orientational polarizability.

\section{Preliminary Relationships}

If a substance has a single macroscopic dielectric relaxation time $\mathbf{T}$, its dielectric behavior in a sinusoidal electric field is described by the Debye [4] equation

$$
\epsilon^{\prime \prime}(\omega)=\frac{\Delta \epsilon \omega \mathbf{T}}{1+\omega^{2} \mathbf{T}^{2}}
$$

where $\epsilon^{\prime \prime}(\omega)$ is the dielectric-loss factor as a function of angular frequency $\omega$, and $\Delta \epsilon$ is the increment of dielectric constant associated with the relaxation process. A plot of $\epsilon^{\prime \prime}$ as a function of $\log _{10} \omega$, using eq (1), yields a narrow and symmetrical loss curve with a maximum at $\omega \mathbf{T}=1$. As has already been indicated, the loss curve observed for many substances is broader than that predicted by the Debye equation. For present purposes, it is convenient to think of such data as being represented by a superposition of Debye-type loss curves, each of which possesses its own relaxation time and magnitude. Therefore, $[1,5]$

$$
\epsilon^{\prime \prime}(\omega)=\sum_{\beta} \frac{\Delta \epsilon_{\beta} \omega \mathbf{T}_{\beta}}{1+\omega^{2} \mathbf{T}_{\beta}^{2}}
$$

where $\mathbf{T}_{\beta}$, with $\beta=1,2, \ldots$, is the set of macroscopic relaxation times, and $\Delta \epsilon_{\beta}$ is the increment of dielectric constant associated with each mode of decay.

Calculations on molecular models of the type to be discussed here characteristically lead to a set of discrete molecular relaxation times $\tau_{\beta}$, and a corre-

\footnotetext{
${ }^{2}$ A misprint in a general formula used in the polarizability calculations occurs in reference [1]. The last term in eq (11), the expression for $\vec{\mu} \cdot \overrightarrow{\mathrm{F}}$, should be cos $\xi_{i}^{\prime} \cos \xi$ and not $\cos \xi_{i}^{\prime} \cos \theta$. The correct expression was employed in making all calculations.
}

sponding set of molecular orientational polarizabilities $\alpha_{\beta}$, where $\beta=1,2, \ldots . A \mathrm{~s}$ in previous papers, it is assumed that the macroscopic and molecular relaxation times are approximately equal, i. e., $\mathbf{T}_{\beta} \cong \tau_{\beta}$, and that each increment of dielectric constant is proportional to the corresponding orienta tional polarizability so that $\Delta \epsilon_{\beta} \cong K \alpha_{\beta}$, where $K$ is a constant. The lack of an exact proportionality in each case is a result of the fact that the electric field at a molecule (internal field) is not identical to the applied field for condensed phases. With the approximations mentioned above we have the working equation $[1,5]$,

$$
\epsilon^{\prime \prime}(\omega) \cong K \sum_{\beta} \frac{\alpha_{\beta} \omega \tau_{\beta}}{1+\omega^{2} \tau_{\beta}^{2}}
$$

for estimating the details of the loss curves. In cases where the quantity $K \Sigma_{\beta} \alpha_{\beta} \cong \Sigma_{\beta} \Delta \epsilon_{\beta} \equiv \epsilon_{s}-\epsilon_{\infty}$ is small $\left(\epsilon_{s}\right.$ and $\epsilon_{\infty}$ are the static and high frequency dielectric constants, respectively), eq (2b) is an excellent approximation. Furthermore, in cases where the Onsager expression for the ratio of the internal to the applied field is valid, there are reasons for believing that eq $(2 \mathrm{~b})$ holds with sufficient accuracy for the purpose at hand for any value of $\epsilon_{s}-\epsilon_{\infty}$ $[5,6,7]$.

All of the types of loss curves mentioned here and in section 1 can be reproduced by inserting the appropriate $\tau_{\beta}$ and $\alpha_{\beta}$ into eq $(2 \mathrm{~b})$. In particular, if two relaxation times (or sets of relaxation times) are very far apart, and the corresponding polarizabilities are of comparable magnitude, a bimodal loss curve is generated.

In the special case where only two active relaxation times exist, we have been able to deduce the following useful condition for the appearance of clearly apparent bimodal loss curves:

$$
\left(\frac{\tau_{l}}{\tau_{s}}\right)\left(\frac{\alpha_{\min }}{\alpha_{\max }}\right)>8 .
$$

In this equation $\tau_{l}$ and $\tau_{s}$ represent the longer and shorter relaxation times, respectively, and $\alpha_{\max }$ and $\alpha_{\mathrm{min}}$ the larger and smaller polarizabilities. Relaxation times and polarizabilities conforming with this equation will yield loss curves that exhibit a perceptible minimum between the two loss regions.

\section{Pear-Shaped Molecules in a Body- Centered Orthorhombic Lattice (0-1, 0-2)}

\subsection{Basic Model}

In this section the manner in which a structure consisting of pear-shaped molecules situated in bco lattice may give rise to a bimodal loss curve is considered. The unit cell of the molecular model to be discussed is shown in figure 1 (a). Each molecule in the lattice is supposed to consist of a large spheroidal part on which a smaller more or less hemispherical protuberance is attached. The protuberance is shown in black in figure 1 (a). The dipole moment $\mu$ is along the line connecting the center of the larger spheroid and the center of the protuberance. 


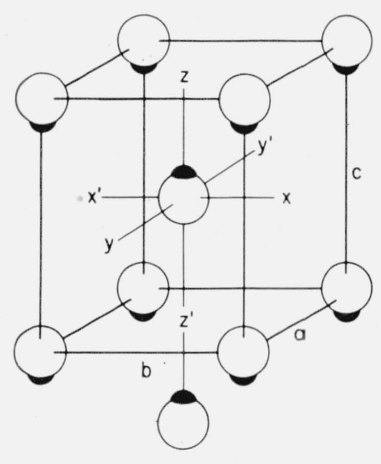

a

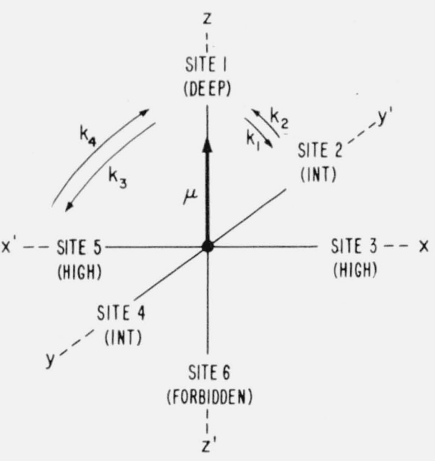

b

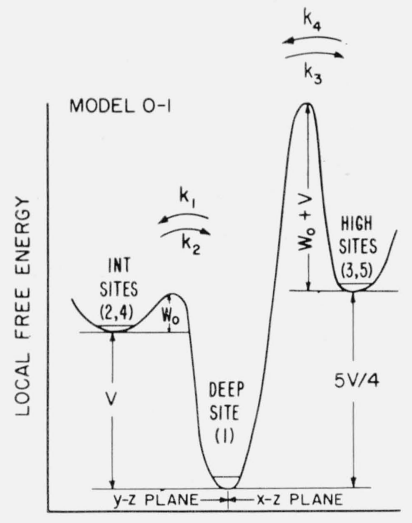

ANGULAR POSITION

C

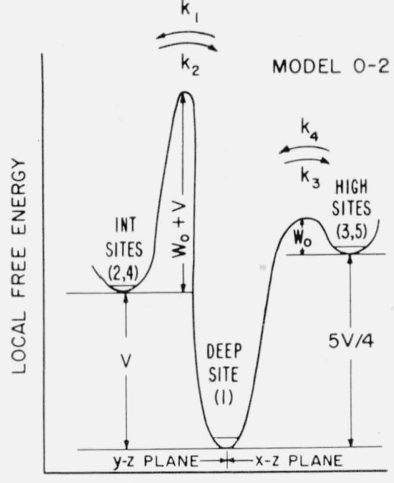

ANGULAR POSITION

d

FIGURE 1. Details of the bco lattice with pear-shaped molecules.

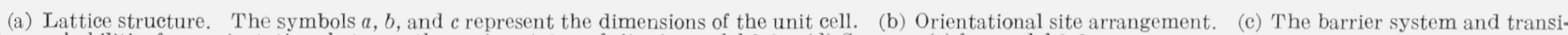
tion probabilities for reorientations between the various types of sites in model $0-1$. (d) Same as (c) for model $0-2$.

The first step is to single out a typical molecule - it is convenient to consider the central one shown in figure 1 (a) - and select a reasonable set of equilibrium orientations (sites) and relative site energies as the central dipole is permitted to rotate with its neighbors held fixed. This will determine many important features of the crystalline field. In the present case, it is postulated that the protuberance on the central dipole may fit between the four nearest neighbors comprising each face of the unit cell so that the possible dipole orientations are orthogonal.

In the face-centered orthorhombic $(f c o)$ structure there are 14 rather than 6 gaps between the nearest neighbors in which the protuberance might conceivably fit. The six most pronounced sites, which are in the directions of the nextnearest neighbors, are orthogonal to one another; thus these sites resemble those postulated for models $0-1$ and $0-2$ (sections 3.2 and 3.3). The eight unstable sites are a result of the protuberance fitting into triads of nearest neighbors. If it is assumed that these eight sites are sufficiently unstable to be ignored, the site model for the fco lattice becomes identical to that for the $b c o$. Under these conditions, the results quoted here for the bco lattice with pear-shaped molecules will hold for the fco lattice as well.

The assumption is made that there is one most stable orientation for the central dipole, and this deep site is designated " 1 ." In the bco lattice, where $a<b<c$ for the unit cell, the equilibrium site energies in the $x$ and $x^{\prime}$ directions (sites 3 and 5) must be the same, and similarly, the pair of sites in the $y$ and $y^{\prime}$ directions (sites 2 and 4) must have the same energy. However, since in this lattice the nearest neighbors in the $a c$ faces are somewhat more compactly arranged than those in the $b c$ faces, the pair of sites 3, 5 is less stable than the pair 2, 4 . Hence, sites 3 and 5 are referred to as "high" sites, and 2 and 4 are designated as "intermediate" sites (fig. 1 (b)). It has been assumed that the dipole cannot point in the $z^{\prime}$ direction owing to the repulsion of the protuberance on the central molecule with that on the body-centered molecule below it; this idea has been borrowed from the work of Powles [8] on molecules of similar shape.
In antiparallel dipole arrays of the type shown in figure 1 (a) where $a \cong b \cong c$, the orienting torque on the central dipole due to the electric field exerted by its neighbors will generally be small. In $b c c$ lattice where $a=b=c$ this torque is actually zero. In such situations it is safe to ignore the effect of dipolar interaction on the site energies, but in strongly tetragonal or orthorhombic unit cells, or certain parallel dipole arrays, an appreciable contribution to the site energies may arise from dipolar interaction.

Many of the equilibrium properties of the site model shown in figure 1 (b) can be obtained without introducing further information. For instance, the total orientational polarizability can be calculated in terms of $\mu$, the absolute temperature, $T$, and the equilibrium energy differences between the sites. However, the relaxation times of interest here depend on the activation energy barriers arising from the crystalline field that control the rate at which dipoles turn from one site to another. The local free energy barrier $W_{i j}$ between two adjacent sites $i$ and $j$ determines the elementary process transition probability (unimolecular rate constant) $k_{i \rightarrow j}$ as $k_{i \rightarrow j}=A \exp \left[-W_{i j} / \boldsymbol{k} T\right]$, where $\boldsymbol{k}$ is Boltzmann's constant, and $A$ a constant. ${ }^{3}$ In a similar manner, $k_{j \rightarrow i}=A \exp \left[-W_{j i} / \boldsymbol{k} T\right]$. In turn, certain sets of combinations of the various transition probabilities prove to be related to the set of relaxation times that characterize the system. In order to obtain the required molecular relaxation times, it is therefore necessary to determine or assume certain properties of the activation barrier system in such a manner as to fix at least the relative values of the various transition probabilities.

Certain simple considerations greatly limit the

${ }^{3}$ In assuming that each transition probability is controlled by the free energy" of activation, we follow the ideas expressed previously by Kauzmann [Rev. Mod. Phys. 14, 12 (1942)] concerning the elementary dipole orientation process in dielectric. In the text, the term "energy", is sometimes used to replace the more cumbersome "local_free energy," especially in discussing the equilibrium properties of a site. 
possible choices of the relative transition probabilities for any model:

(a) The only elementary process is the turning of a dipole to an adjacent site (single-jump hypothesis, $S-J)$. The $S-J$ hypothesis has been used in all previous calculations $[1,2,3,5]$.

(b) If $i$ and $j$ are adjacent sites of equivalent equilibrium energy, then $k_{i \rightarrow j}=k_{j \rightarrow i}$ as the activation barrier is the same for each process. This also follows from the principle of detailed balance.

(c) Let the probability that a dipole will turn from a deep site to a high site be denoted $k_{\text {deep } \rightarrow \text { high }}$, and the probability for the corresponding reverse process be $k_{\text {high } \rightarrow \text { deep. }}$ It follows from the fact that the activation barrier for the process deep $\rightarrow$ high must be greater than that for the process high $\rightarrow$ deep that $k_{\text {deep } \rightarrow \text { high }}<k_{\text {high } \rightarrow \text { deeep }}$.

(d) The transition probabilities for the turning of a dipole from a certain site to any of a number of adjacent sites that possess equivalent equilibrium energy must be identical for reasons of symmetry of the molecule and of the crystal lattice.

Even with the above, the general orthorhombic model with pear-shaped molecules is excessively complicated and difficult to deal with from a mathematical standpoint. Hence we have introduced the assumption that reorientations in the $x-y$ plane are much more difficult to make as elementary processes than other allowed transitions, i. e., $k_{x-y} \ll k_{x-z}$ or $k_{y-z}$; this condition merely requires $W_{x-y}$ to be a few times $W_{x-z}$ and $W_{y-z}{ }^{4}$ Then for purposes of calculation we pass to the limit and set $k_{x-y}$ equal to zero. As a result of this assumption and subsequent limiting procedure, processes of the type $2 \rightarrow 3$ are forbidden, but it should be noted that a dipole may turn from one site to another in the $x-y$ plane as the result of a succession of elementary processes by first entering site 1 . The above-mentioned simplification was first introduced to render the problem more tractable, but further considerations, discussed below, suggest that this condition is physically not unreasonable under certain circumstances.

Assume that the repulsion between two protuberances virtually in contact is more severe than that between the body of the molecule and a protuberance under the same conditions. First, note that this assumption is consistent with the forbidding of site 6 as a possible orientation. Second, in turning the protuberance on the central molecule in the $x-y$ plane, observe (fig. 1 (a)) that when it is turned half-way to the next site it comes into close contact at a right angle with the protuberance on a nearest neighbor, thus leading to a situation much like that used to forbid site 6 . It would, therefore, appear to be permissible on a trial basis to forbid this activated state and hence elementary processes in the $x-y$ plane. Note finally that the stated repulsion hypothesis permits the necessary elementary processes between the sites in the $x-y$ plane and site 1 . It was not found possible to justify clearly the forbidding of reorientations in the $x-y$ plane on

\footnotetext{
${ }_{4}$ The assumption that $\left.\left.k_{x y}\right)\right\rangle k_{x z}$ or $k_{y z}$ will also lead to "bimodal loss "curves for the bco lattice (see section 3.4 ).
}

the basis of a simple "hard sphere" model without introducing special shapes for the protuberance.

The "hard sphere" model is useful in indicating possible sites. However, we find this concept is not very helpful as a guide for determining relative site energies and transition probabilities. For reasons of simplicity, suppose the neighbors are held fixed while the central molecule is reoriented. Then with the simplest approximation the following is found: first, either an orientational site is possible, or there is interference and it is not allowed; second, all allowed sites have the same energy; and third, with regard to the barrier heights, either a transition is allowed and the associated barrier negligible, or the transition is forbidden and the energy barrier is infinitely high. As a second approximation in using the "hard sphere" or rigid, incompressible molecule picture, it might be assumed that a site is not forbidden if a small overlap occurs, but rather that the site energies relative to the most stable site are indicated roughly by the degree of overlap and, similarly, the heights of the free energy barriers that must be surmounted are indicated by the amount of volume overlap. The second approximation could be modified by permitting the neighbors to be displaced and to rotate slightly when the central molecule is reoriented, a probably more realistic representation than that previously assumed. However, this relaxation of perfect crystalline structure would greatly complicate the problem.

An alternative to the "hard sphere" method of estimating equilibrium orientations and activation barriers is to use a potential law of the form $u(r)=-A / r^{6}+B / r^{n}$, where different values of $A, B$, and $n$ are assigned to the body of the molecule and the protuberance. Such calculations are hampered by a lack of information concerning the exact repulsive law (i. e., the value of $n$ ) that should be used. In addition, if directional attractive forces such as evidently exist in the hydrogen bond are present, this further complicates the situation. Nevertheless, it is believed that if further mathematical labor is to be invested in this problem, it will probably be most profitable to employ a method that involves attractive and repulsive potentials which vary with distance.

Within the limits imposed by items (a) through (d) above and the absence of elementary processes between sites in the $x-y$ plane, two basic types of barrier system are still possible for the postulated arrangement of sites and equilibrium energies. The first type, denoted 0-1, has a smaller barrier for jumps of the type intermediate $\rightarrow$ deep than for high $\rightarrow$ deep, while the second, 0-2, has a larger barrier for intermediate $\rightarrow$ deep than for high $\rightarrow$ deep. Specific examples of these two types are considered separately below.

\subsection{Model 0-1}

The simplest way to study the properties of the $b c o$ lattice with pear-shaped molecules for the special case where the barrier for the process intermediate $\rightarrow$ deep is smaller than that for the process high $\rightarrow$ deep is with the help of a specific example. The specific barrier system chosen to illustrate the behavior of this model is shown in figure 1 (c). As has been noted previously for the orthorhombic lattice, the sites in the $x-y$ plane must, taken pairwise, differ in equilibrium energy. Hence the intermediate sites are taken to have an energy $V$ above the deep site, while the high sites are set at $5 \mathrm{~V} / 4$ above the deep site. Although these assignments are arbitrary, it is emphasized that similar results are obtained for models where the equilibrium energy of the high sites is set in the range $V$ to roughly $2 V$ above the deep site. The model is so arranged that as $V$ is taken to be zero, all barriers have the value $W_{0}$. 
The transition probabilities for the elementary processes are defined as follows:

$$
\left.\begin{array}{l}
k_{\text {deep } \rightarrow \text { int. }}=k_{1}=A e^{-\left(W_{0}+V\right) / \boldsymbol{k} T} \\
k_{\text {int. } \rightarrow \text { deep }}=k_{2}=A e^{-W_{0} / \boldsymbol{k} T} \\
k_{\text {deep } \rightarrow \text { high }}=k_{3}=A e^{-\left(9 V / 4+W_{0}\right) / \boldsymbol{k} T} \\
k_{\text {high } \rightarrow \text { deep }}=k_{4}=k_{1}=A e^{-\left(W_{0}+V\right) / k T}
\end{array}\right\}
$$

As a result of the aforementioned simplification regarding reorientations in the $x-y$ plane, $k_{\text {int. } \rightarrow \text { high }}=$ $k_{\mathrm{h} \text { igh } \rightarrow \text { int. }}=0$. It is noted further that the assignments given in eq (4) conform with considerations (c) and (d) mentioned in section 3.1, page 62. In accord with (c) $k_{2}>k_{1}$ and $k_{4}>k_{3}$, and in accord with (d) the same transition probability is used to describe the elementary processes occurring between the paired sites of equivalent energy and the deep site, e. g., $k_{13}=k_{15}=k_{\text {deep } \rightarrow \text { high. }}$ Note also that $k_{1}=k_{4}$ as the corresponding barriers are identical in the model. Item (b) does not apply in this particular model be- cause there are no adjacent sites with the same equilibrium energy.

It is now a simple matter to write down the differential equations that govern the rate processes. Let $N_{1}, N_{2}, \ldots$. represent the number of dipoles in sites $1,2, \ldots$ at any time $t$. Then, bearing in mind the $S-J$ hypothesis, which forbids jumps of the type $3 \rightarrow 5$ or $2 \rightarrow 4$ as an elementary process, the net rates at which dipoles enter and leave each site are

$$
\left.\begin{array}{l}
d N_{1} / d t=-2\left(k_{1}+k_{3}\right) N_{1}+k_{2} N_{2}+k_{4} N_{3}+k_{2} N_{4}+k_{4} N_{5} \\
d N_{2} / d t=-k_{2} N_{2}+k_{1} N_{1} \\
d N_{3} / d t=-k_{4} N_{3}+k_{3} N_{1} \\
d N_{4} / d t=-k_{2} N_{4}+k_{1} N_{1} \\
d N_{5} / d t=-k_{4} N_{5}+k_{3} N_{1}
\end{array}\right\}
$$

The relaxation times may readily be obtained by solving the above equations using the methods given in previous papers and the polarizabilities ${ }^{5}$ may be obtained by using the method outlined in reference [1].

The results for $0-1$ are as follows:

$$
\begin{aligned}
\tau_{2} & =1 / k_{2}, \alpha_{2}=\frac{2 g}{\left(1+2 g+2 g^{5 / 4}\right)}\left(\frac{\mu^{2}}{3 \boldsymbol{k} T}\right) ; \\
\tau_{3} & =1 / g k_{2}, \alpha_{3}=\frac{2 g^{5 / 4}}{\left(1+2 g+2 g^{5 / 4}\right)}\left(\frac{\mu^{2}}{3 \boldsymbol{k} T}\right) ; \\
\tau_{4} & =\frac{2}{k_{2}\left(1+3 g+2 g^{9 / 4}+\sqrt{q}\right)}, \\
\alpha_{4} & =\frac{-\left(1+g+4 g^{5 / 4}-2 g^{9 / 4}+\sqrt{q}\right)\left(1-3 g-2 g^{9 / 4}-\sqrt{q}\right)}{4\left(1+2 g+2 g^{5 / 4}\right)^{2} \sqrt{q}}\left(\frac{\mu^{2}}{3 \boldsymbol{k} T}\right) ; \\
\tau_{5} & =\frac{2}{k_{2}\left(1+3 g+2 g^{9 / 4}-\sqrt{q}\right)}, \\
\alpha_{5} & =\frac{\left(1+g+4 g^{5 / 4}-2 g^{9 / 4}-\sqrt{q}\right)\left(1-3 g-2 g^{9 / 4}+\sqrt{q}\right)}{4\left(1+2 g+2 g^{5 / 4}\right)^{2} \sqrt{q}}\left(\frac{\mu^{2}}{3 \boldsymbol{k} T}\right) ; \\
\alpha_{\text {total }} & =\left[1-\frac{1}{\left(1+2 g+2 g^{5 / 4}\right)^{2}}\right]\left(\frac{\mu^{2}}{3 \boldsymbol{k} T}\right) .
\end{aligned}
$$

In the above equations $g=k_{1} / k_{2}=e^{-V / k T}$ and $q=$ $1+2 g+g^{2}-4 g^{9 / 4}+12 g^{13 / 4}+4 g^{9 / 2}$.

The details of the loss curves for this model are most readily ascertained by calculating the orientational polarizabilities and relaxation times numerically from eq (6) for various values of the parameter $g=\exp (-V / \boldsymbol{k} T)$, inserting these in eq $(2 \mathrm{~b})$, and plotting the loss curves. The relevant data are given in table 1 , and the loss curves obtained from these data illustrated in figure 2.
It is seen that for small values of $g$ a strongly bimodal loss curve is produced which is characterized by a high-frequency peak which is larger than the low-frequency one. Both loss maxima are actually composed of two distinct peaks which are very close together with the result that, considered individually, each of the two loss peaks is very nearly of the Debye type. As $g$ increases the peaks tend to come closer

${ }_{5}$ The polarizabilities given in the paper are derived on the assumption that the medium is polycrystalline. 
TABLE 1. Relaxation times and polarizabilities for model 0-1

\begin{tabular}{|c|c|c|c|c|c|c|c|c|c|}
\hline \multirow{2}{*}{$g$} & \multicolumn{5}{|c|}{ Orientational polarizabilities a } & \multicolumn{4}{|c|}{ Relaxation times b } \\
\hline & $\alpha_{2}$ & $\alpha_{3}$ & $\alpha_{4}$ & $\alpha_{5}$ & $\alpha_{\text {total }}$ & $\tau_{2}$ & $\tau_{3}$ & $\tau_{4}$ & $\tau_{5}$ \\
\hline $\begin{array}{l}1 \\
0.1 \\
.01 \\
.001\end{array}$ & $\begin{array}{l}0.4 \\
.1524 \\
.01949 \\
.001995\end{array}$ & $\begin{array}{l}0.4 \\
.0857 \\
.00616 \\
.000355\end{array}$ & $\begin{array}{l}0.16 \\
.1292 \\
.01911 \\
.001992\end{array}$ & $\begin{array}{l}0 \\
.0522 \\
.00588 \\
.000353\end{array}$ & $\begin{array}{l}0.96 \\
.4195 \\
.05064 \\
.004695\end{array}$ & $\begin{array}{l}1 \\
1 \\
1 \\
1\end{array}$ & $\begin{array}{r}1 \\
10 \\
100 \\
1000\end{array}$ & $\begin{array}{r}0.20 \\
.83 \\
.98 \\
1\end{array}$ & $\begin{array}{c}1 \\
9.2 \\
99.4 \\
1000\end{array}$ \\
\hline
\end{tabular}

a In units of $\mu^{2} / 3 \boldsymbol{k} t$.

b In units of $1 / \boldsymbol{k}_{2}=1 / A e^{-W_{0} / \boldsymbol{k} T}$.

together and finally coalesce, but even at $g=1$ the loss curve is still somewhat broader than a Debyetype peak. As may be seen from the expression $g=\exp (-V / \boldsymbol{k} T)$, the trends just noted for increasing $g$ hold as well for the case of rising temperature.

To the approximation used here, the static dielectric constant is given by $\epsilon_{s} \cong \epsilon_{\infty}+K \alpha_{\text {total }}$, where $\alpha_{\text {total }}=$ $\Sigma_{\beta} \alpha_{\beta}$. A simple analysis shows that $\alpha_{\text {total }}$ (and hence $\epsilon_{s}$ ) will rise with increasing temperature until $g$ reaches a value of 0.17 ; at this point the explicitlyshown $1 / T$ term in the expression for $\alpha_{\text {total }}$ in eq (6) overcomes the increase with temperature of the term containing $g$ inside the brackets. Thus $d \epsilon_{s} / d T$ is positive for $0<g<0.17$ and negative for $0.17<g \leq 1$. It should be noted that the loss curves are strongly bimodal only in the region $0<g<0.06$, i. e., where $d \epsilon_{s} / d T$ is positive, and conversely, that only a somewhat broadened loss curve is obtained when $d \epsilon_{s} / d T$ has a strongly negative value, as for $g \cong 1$.

\subsection{Model 0-2}

This is the model shown in figure 1 (d) where the activation energy barrier for reorientations from high $\rightarrow$ deep is less than that for intermediate $\rightarrow$ deep. For this case the transition probabilities are defined as follows:

$$
\left.\begin{array}{l}
k_{\text {deep } \rightarrow \text { int. }}=k_{1}=A e^{-\left(W_{0}+2 V\right) / k T} \\
k_{\text {int. } \rightarrow \text { deep }}=k_{2}=A e^{-\left(W_{0}+V\right) / k T} \\
k_{\text {deep } \rightarrow \text { high }}=k_{3}=A e^{-\left(W_{0}+5 V / 4\right) / k T} \\
k_{\text {high } \rightarrow \text { deep }}=k_{4}=A e^{-W_{0} / k T .}
\end{array}\right\}
$$

It will be noted that the assignments listed above are consistent with the considerations regarding the relative values of the transition probabilities mentioned in section 3.1. The differential equations are identical to those given for $0-1$, except that eq (7) is used rather than eq (4) to define the transition probabilities. The relaxation times and polarizabilities are found to be
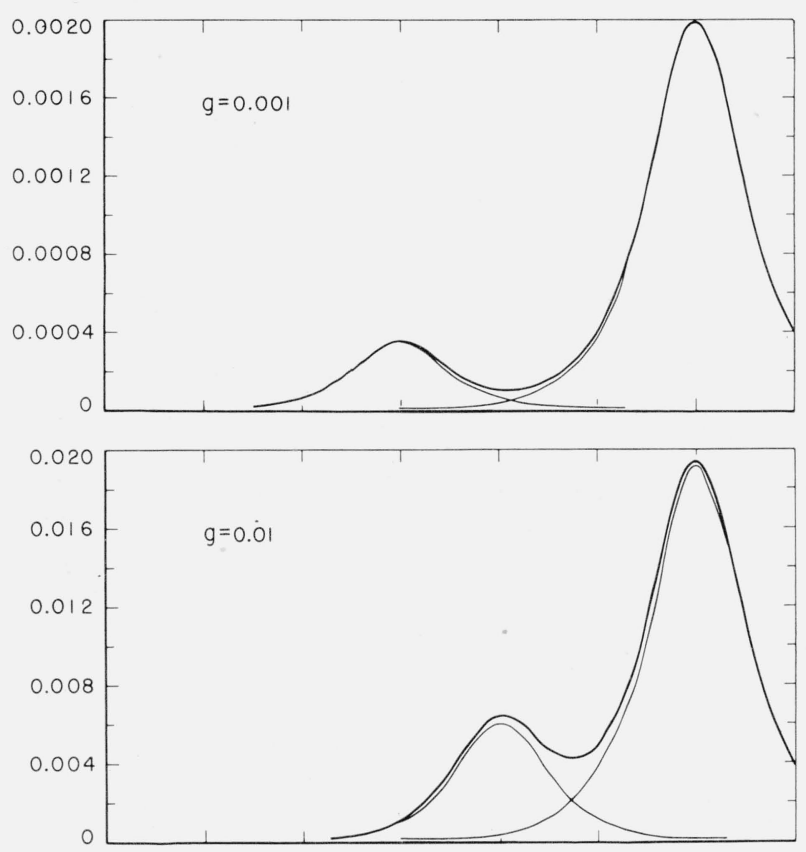

iv
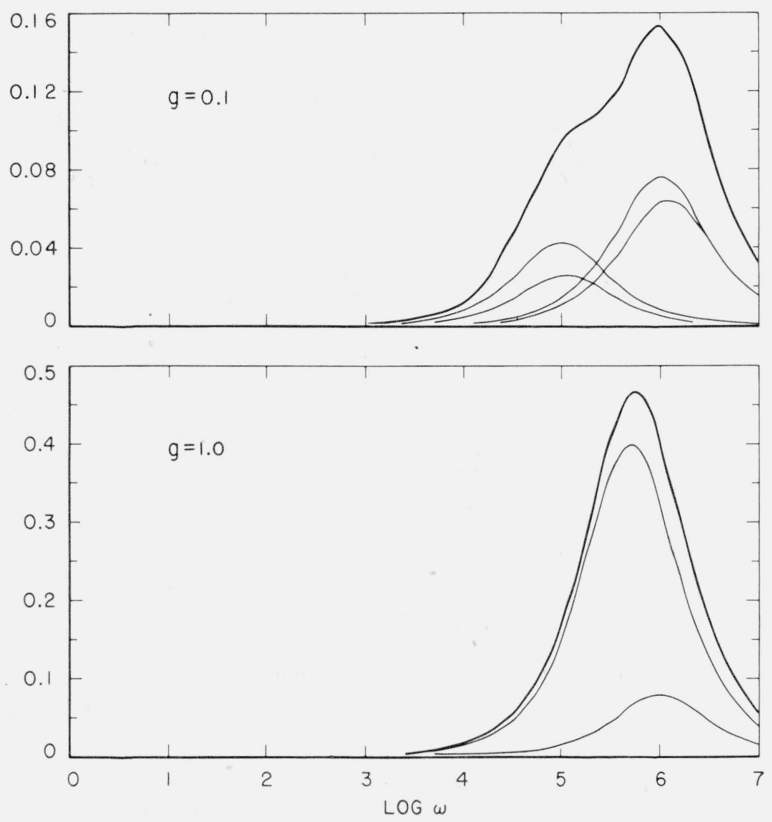

FiguRE 2. Shape of the dielectric loss curves for model 0-1 for various values of $g$.

The loss factor $\epsilon^{\prime \prime}$ is given with $\mathrm{K}\left(\mu^{2} / 3 \boldsymbol{k} T\right)$ normalized to unity. The shortest relaxation time has been set at $10^{-6}$ sec. in each diagram. The component loss peaks associated with each relaxation time are shown as light lines and the result-
ant loss curve is the heavy line. 


$$
\begin{aligned}
& \tau_{2}=1 / k_{4} g, \alpha_{2}=\frac{2 g}{1+2 g+2 g^{5 / 4}}\left(\frac{\mu^{2}}{3 \boldsymbol{k} T}\right) ; \\
& \tau_{3}=1 / k_{4}, \alpha_{3}=\frac{2 g^{5 / 4}}{1+2 g+2 g^{5 / 4}}\left(\frac{\mu^{2}}{3 \boldsymbol{k} T}\right) ; \\
& \tau_{4}=\frac{2}{k_{4}\left(1+g+2 g^{2}+2 g^{5 / 4}+\sqrt{r}\right.}, \\
& \alpha_{4}=\frac{-\left[2 g\left(1+2 g+2 g^{5 / 4}\right)-j+\sqrt{r}\right][-j-\sqrt{r}+2 g]}{4 g\left(1+2 g+2 g^{5 / 4}\right)^{2} \sqrt{r}}\left(\frac{\mu^{2}}{3 \boldsymbol{k} T}\right) ; \\
& \tau_{5}=\frac{2}{k_{4}\left[1+g+2 g^{2}+2 g^{5 / 4}-\sqrt{r}\right]}, \\
& \alpha_{5}=\frac{\left[2 g\left(1+2 g+2 g^{5 / 4}\right)-j-\sqrt{r}\right][-j+\sqrt{r}+2 g]}{4 g\left(1+2 g+2 g^{5 / 4}\right)^{2} \sqrt{r}}\left(\frac{\mu^{2}}{3 \boldsymbol{k} T}\right) ; \\
& \alpha_{\text {total }}=\left[1-\frac{1}{\left(1+2 g+2 g^{5 / 4}\right)^{2}}\right]\left(\frac{\mu^{2}}{3 \boldsymbol{k} T}\right),
\end{aligned}
$$

where $g=k_{2} / k_{4}=e^{-V / k T}, r=1-2 g-3 g^{2}+4 g^{3}+4 g^{4}+$

$$
4 g^{5 / 4}\left(1-g+g^{5 / 4}+2 g^{2}\right) \text {, and } j=1+g+2 g^{2}+2 g^{5 / 4} .
$$

Values of the polarizabilities and relaxation times computed with eq (8) are given in table 2. The loss curves calculated for some of the $g$ values are plotted in figure 3.

'It is seen that this model leads to strongly bimodal loss curves which differ from those obtained with model $0-1$ primarily in that the largest loss peak is now on the low frequency side. Noting that $g=$ $\exp (-V / \boldsymbol{k} T)$, it is seen that the two loss peaks converge with rising temperature and coalesce into a single broadened and asymmetric peak, which then

\begin{tabular}{|c|c|c|c|c|c|c|c|c|c|}
\hline \multirow{2}{*}{$g$} & \multicolumn{5}{|c|}{ Orientational polarizabilities \& } & \multicolumn{4}{|c|}{ Relaxation times b } \\
\hline & $\alpha_{2}$ & $\alpha_{3}$ & $\alpha_{4}$ & $\alpha_{5}$ & $\alpha_{\text {total }}$ & $\tau_{2}$ & $\tau_{3}$ & $\tau_{4}$ & $\tau_{5}$ \\
\hline $\begin{array}{l}1 \\
0.1 \\
.01 \\
.001\end{array}$ & $\begin{array}{l}0.4 \\
.1524 \\
.01949 \\
.001994\end{array}$ & $\begin{array}{l}0.4 \\
.0857 \\
.00616 \\
.000355\end{array}$ & $\begin{array}{l}0.16 \\
.0798 \\
.00612 \\
.000355\end{array}$ & $\begin{array}{l}0 \\
.1016 \\
.01886 \\
.001992\end{array}$ & $\begin{array}{l}0.96 \\
.4195 \\
.05064 \\
.004695\end{array}$ & $\begin{array}{r}1 \\
10 \\
100 \\
1000\end{array}$ & $\begin{array}{l}1 \\
1 \\
1 \\
1\end{array}$ & $\begin{array}{r}0.20 \\
.871 \\
.996 \\
1.000\end{array}$ & $\begin{array}{c}1 \\
8.49 \\
98 \\
998\end{array}$ \\
\hline
\end{tabular}
becomes narrower with a further increase of temperature. The total polarizability varies with $g$ in a manner identical to that found for $0-1$, with the result that the loss curves are strongly bimodal only in the region where the dielectric constant is low and $d \epsilon_{s} / d T$ positive.

TABLE 2. Relaxation times and polarizabilities for model 0-2

a In units of $\mu^{2} / 3 \boldsymbol{k} t$.

b In units of $1 / \boldsymbol{k}_{2}=1 / A e^{-W_{0} / \boldsymbol{k} T}$.
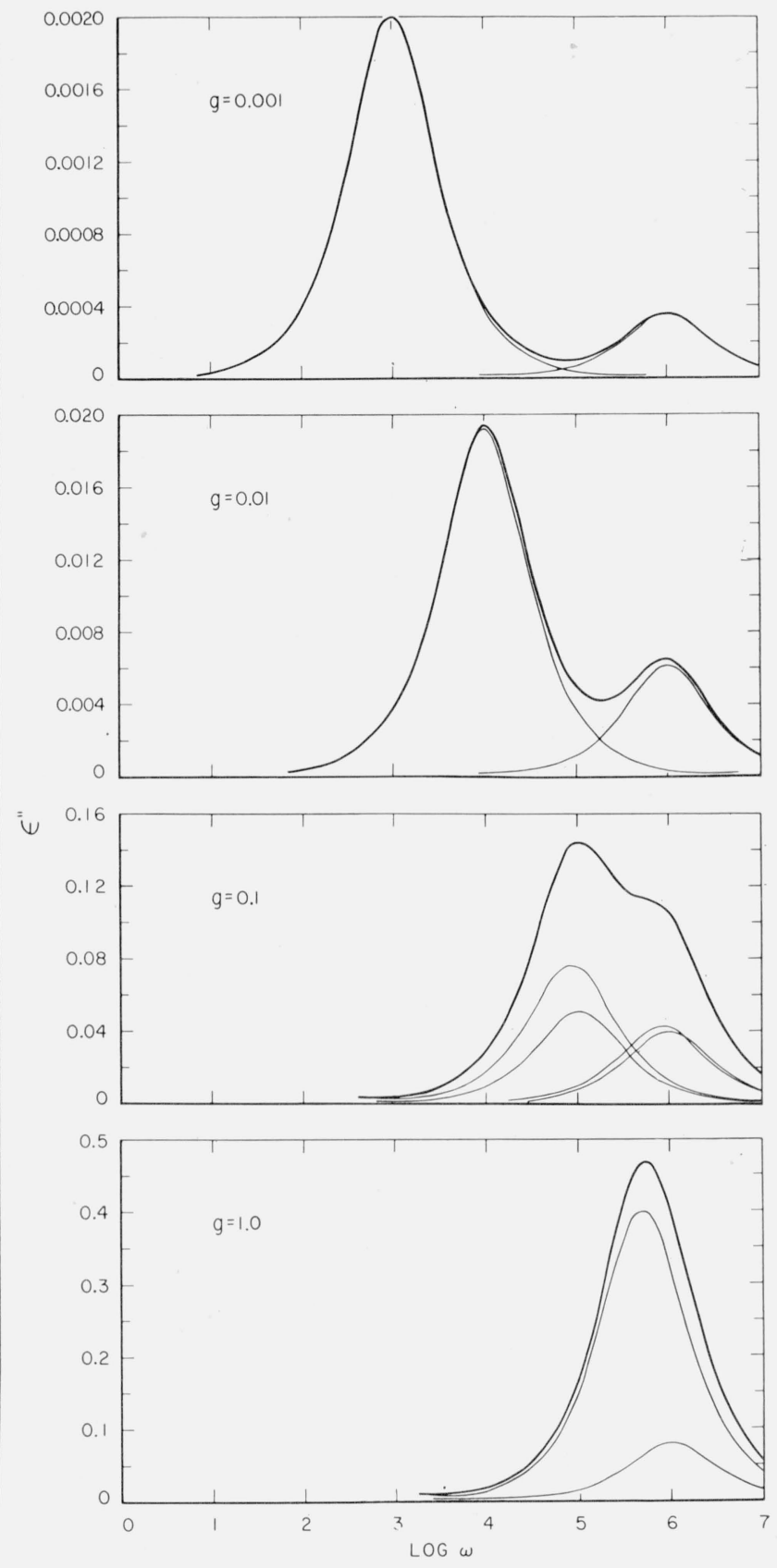

Figure 3. Shape of the dielectric-loss curves for model 0-2 for various values of $g$. 


\subsection{Alternative Approximation Leading to Bimodal Loss Curves in $B C O$ Systems}

At this stage, it is of interest to point out that another type of approximation concerning elementary processes in the $x-y$ plane will lead to bimodal behavior for pear-shaped molecules in the bco lattice. As will be seen in section 4, model T-1, bimodal loss curves can be obtained for pear-shaped molecules in. the bct lattice by permitting reorientations in the $x-y$ plane to be more rapid than for all other elementary processes. It can be shown for the analogous bco case where reorientations in the $x-y$ plane are very rapid compared with the other possible elementary processes that bimodal loss curves are generated. Detailed calculations are not given, but this result is readily seen for the limiting case where the $b c o$ lattice is assumed to be nearly tetragonal so that results closely similar to those quoted for model T-1 are obtained. Bimodal loss curves can be obtained with this approximation which have peaks of equal magnitude, or alternatively, similar to those calculated for O-1. Loss curves with the type of symmetry depicted for O-2 cannot be obtained when it is assumed that reorientations in the $x-y$ plane are more rapid than all other elementary processes.

It is clear from the foregoing that bimodal behavior can arise in the $b c o$ lattice with pear-shaped molecules under widely different circumstances. While it is at present impossible to state with any certainty which of the two approximations (very fast or very slow reorientations in the $x-y$ plane) will hold for any particular real system, it does seem reasonable to suppose that either one or the other should be valid for some substances. It is therefore considered possible on theoretical grounds that some real systems consisting of pear-shaped molecules in the bco lattice will exhibit bimodal behavior, particularly at low temperatures.

\section{Pear-Shaped Molecules in a Body- Centered Tetragonal Lattice (T-1)}

In a search for lattice models that led to bimodal loss curves, pear-shaped molecules in a bct lattice were naturally considered. The molecular model is essentially the same as that depicted in figure 1(a) except that $a=b<c$. Following the type of argument given in section. 3 , one obtains the site energies and orientations shown in figure 4 . In the bct lattice, all of the sites in the $x-y$ plane have the same energy and are designated "high" sites. In order to carry out a general treatment of this model let

$$
\left.\begin{array}{l}
k_{\text {deep } \rightarrow \text { high }}=k \\
k_{\text {high } \rightarrow \text { deep }}=k^{\prime} \\
k_{\text {high } \rightarrow \text { high }}=k^{\prime \prime} .
\end{array}\right\}
$$

Because site 1 is deep, $k^{\prime}>k$, but the transition probability for reorientations between the equivalent high sites, $k^{\prime \prime}$, may in the general mathematical model have any value.

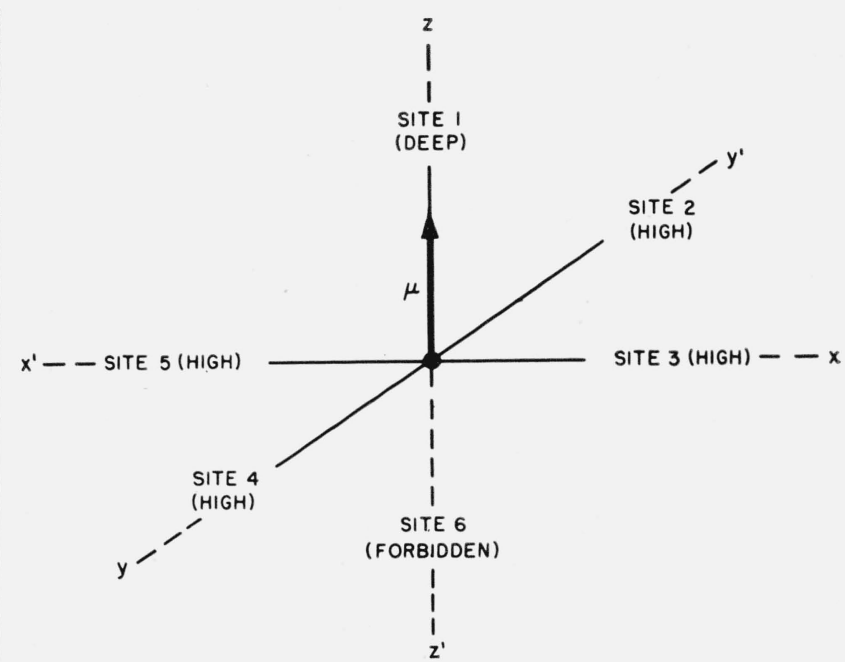

FiguRE 4. Orientational site diagram for pear-shaped molecules in the bct lattice.

The rate equations are

$$
\left.\begin{array}{l}
\frac{d N_{1}}{d t}=-4 k N_{1}+k^{\prime}\left(N_{2}+N_{3}+N_{4}+N_{5}\right) \\
\frac{d N_{2}}{d t}=-\left(k^{\prime}+2 k^{\prime \prime}\right) N_{2}+k N_{1}+k^{\prime \prime}\left(N_{3}+N_{5}\right) \\
\frac{d N_{3}}{d t}=-\left(k^{\prime}+2 k^{\prime \prime}\right) N_{3}+k N_{1}+k^{\prime \prime}\left(N_{2}+N_{4}\right) \\
\frac{d N_{4}}{d t}=-\left(k^{\prime}+2 k^{\prime \prime}\right) N_{4}+k N_{1}+k^{\prime \prime}\left(N_{3}+N_{5}\right) \\
\frac{d N_{5}}{d t}=-\left(k^{\prime}+2 k^{\prime \prime}\right) N_{5}+k N_{1}+k^{\prime \prime}\left(N_{2}+N_{4}\right) .
\end{array}\right\}
$$

The relaxation times are found to be

$$
\left.\begin{array}{l}
\tau_{2}=\tau_{3}=\frac{1}{k^{\prime}+2 k^{\prime \prime}} \\
\tau_{4}=\frac{1}{4 k^{\prime \prime}+k^{\prime}} \\
\tau_{5}=\frac{1}{4 k+k^{\prime}} .
\end{array}\right\}
$$

The corresponding polarizabilities are

$$
\left.\begin{array}{l}
\alpha_{2}+\alpha_{3}=\frac{4 g}{1+4 g}\left(\frac{\mu^{2}}{3 \boldsymbol{k} T}\right) \\
\alpha_{4}=0 \\
\alpha_{5}=\frac{4 g}{(1+4 g)^{2}}\left(\frac{\mu^{2}}{3 \boldsymbol{k} T}\right) \\
\alpha_{\mathrm{t} \text { tal }}=\frac{8 g(1+2 g)}{(1+4 g)^{2}}\left(\frac{\mu^{2}}{3 \boldsymbol{k} T}\right)
\end{array}\right\}
$$


with $g=k / k^{\prime}=e^{-V / k T}$, where $V$ is the equilibrium energy difference between the deep and high sites. Mode 4 is inactive in the dielectric relaxation spectrum owing to a compensatory motion of the dipoles.

Remembering that $k^{\prime}>k$, and that $\tau_{4}$ is inactive, it is seen from eq (11) that the active relaxation times are always close together except for the case $k^{\prime \prime} \gg k, k^{\prime}, i$. e., where the transitions in the $x-y$ plane are considerably more rapid than all other elementary processes. It is worth while to consider the nature of the loss curves for small values of $g$ (ordered lattice with low dielectric constant) and values of $g$ close to unity (disordered lattice with liquidlike dielectric constant). When $k^{\prime \prime} \gg k, k^{\prime}$, and $g$ is small, two widely separated loss curves of almost identical magnitude are obtained in contrast to the unequal peaks obtained for models $0-1$ and $0-2$. On the other hand, if the lattice is rotationally disordered $(g \cong 1)$ so that all of the sites have nearly the same equilibrium energy, it would appear to be unreasonable to assume generally that the activation barriers will differ greatly. Hence, in a disordered lattice of the type considered, the condition $k^{\prime \prime} \gg k, k^{\prime}$ may be regarded at least tentatively as being physically unlikely with the result that bimodal behavior is not ordinarily to be expected in such a system.

It may be concluded that pear-shaped molecules in the bct lattice will exhibit bimodal behavior provided the condition $k^{\prime \prime} \gg k, k^{\prime}$ is attained. Such a condition is most likely to arise in. an ordered phase where the dielectric constant is relatively low and $d \epsilon_{s} / d T$ positive. Thus, whereas the $b c o$ lattice yields bimodal loss curve with either very rapid or very slow reorientations in the $x-y$ plane, the corresponding effect can be obtained for the bct lattice only when transitions in the $x-y$ plane are very rapid.

As may be seen from the expression $k_{i j}=A$ exp $\left(-W_{i j} / \boldsymbol{k} T\right)$, the quantities $k, k^{\prime}$, and $k^{\prime \prime}$ will tend to converge with rising temperature. Thus, the two loss peaks comprising a bimodal loss curve will tend to converge as the temperature is raised. As may be seen from a comparison of the quantities $\alpha_{2}+\alpha_{3}$ and $\alpha_{5}$, the two loss peaks in a bimodal curve will be of very nearly the same magnitude when $g$ is small, but as $g$ is increased, i. e., as the temperature is increased, the peak associated with $\tau_{2,3}$ becomes larger than that associated with $\tau_{5}$.

The results for the $b c c$ lattice may be obtained by passing to the limit $g=1$ for the model discussed above. For this highly isotropic lattice it is considered improbable that the condition $k^{\prime \prime} \gg k, k^{\prime}$ could hold generally, and it is therefore anticipated that bimodal loss curves will not commonly arise in such systems. The behavior of the static dielectric constant for such a system will resemble that of a polar liquid, i. e., $\epsilon_{s}$ will be relatively large and $d \epsilon_{s} / d T$ strongly negative.

\section{Discussion}

\subsection{General}

It has been demonstrated that, given certain stated conditions that depend on the details of the crystal- line field, bimodal loss curves can arise in systems consisting of polar three-dimensional rotators with assumed equilibrium orientations and site energies that are consistent with certain types of lattice structure and molecular shape. The basic cause of the set of relaxation times is the anisotropy of the crystalline field in which the molecules are situated. This anisotropy gives rise to different temperaturedependent transition probabilities (unimolecular rate constants) for reorientations between adjacent sites, and the equations describing the net rate at which dipoles enter and leave each site in terms of these transition probabilities then lead in a natural way to the set of discrete relaxation times. Only a single or narrowly spaced set of relaxation times appears if the transition probabilities are all identical as is assumed for a highly isotropic lattice. The relaxation times are generally most widely separated in a lattice with a high degree of anisotropy.

It appears from these studies that rather special forms of the crystalline field are required to produce bimodal behavior. Even after postulating reasonable equilibrium orientations and relative site energies, and applying the rules outlined in section 3.1 concerning the permissible range of transition probabilities, it was generally found that parameters concerned with the nature of certain activated states remained. It will be recalled that at this stage it was noted that particular choices of these parameters led to the widely divergent relaxation times necessary for bimodal behavior. We emphasize that it was generally difficult to really justify these particular choices on the basis of lattice structure, molecular shape, or special types of interactions. Therefore, despite the fact that the above-mentioned special choices concerning the height (or relative height) of the activation energy barrier between certain sites did not seem to be excluded for any physical reason, it was impossible to state with any certainty whether any real system with the designated crystal structure and molecular shape would necessarily show bimodal behavior. It should be remarked that the conclusion that highly disordered (isotropic) lattices will not in general exhibit bimodal behavior rests on the validity of the idea that in a lattice with equivalent equilibrium site energies, it is improbable that the barriers between the various sites will differ greatly.

It is interesting to note that the general molecular explanation for bimodal loss curves in polycrystalline specimens is consistent with the suggestion of Cole and coworkers $[9,10,11,12]$ that the bimodal effect is associated with the dielectric anisotropy of each single crystallite. In their view, the polarization of a single crystal of a substance with a bimodal loss curve should probably be described by two or three principal dielectric constants, some of which may be presumed to decay after the abrupt removal of the electric field at a different net rate. As may be seen especially in earlier papers $[1,3,5]$, this is exactly the situation that prevails for single crystals in the present type of theory. In the process of calculating the orientational polarizabilities, the polarization for each mode of decay was resolved in the $x, y$, and $z$ directions, and it was always found that a given 
mode was much stronger along one or two of these axes. This holds for all of the models leading to the bimodal effect investigated in this paper.

\subsection{Effect of Temperature on the Dielectric Relaxa- tion Spectrum}

In common with almost all of the models studied earlier $[1,3,5]$ the relaxation times converge with increasing temperature. The fundamental reason for this is that each of the set of relaxation times is given by the theory in terms of elementary process transition probabilities defined as $k_{i j}=A \exp \left(-W_{i j}\right)$ $\boldsymbol{k} T)$; clearly the $k_{i j}$ must converge with increasing temperature, and noting that the theory usually shows that the active relaxation times converge as the $k_{i j}$ converge, the result quoted above follows. As a natural result of the convergence of the active dielectric relaxation times, the two loss peaks in a bimodal loss curve tend to merge with rising temperature. If the loss is confined to a single but somewhat broadened peak, the peak will tend to narrow further with rising temperature. It will be noticed for the models treated that, as a result of the special way in which the activation barriers were defined, it was possible to express the relaxation times in terms of the parameter $g=\exp (-V / \boldsymbol{k} T)$. Noting that the active relaxation times for these models always converge as $g$ goes from zero to unity, and remembering that $V$ is the equilibrium energy difference, the convergence of the relaxation times with increasing temperature in these specific cases may be regarded as a direct consequence of the Maxwell-Boltzmann distribution law.

An analysis of the shift with frequency of the two loss peaks in a bimodal loss curve as a function of temperature for all the models studied indicates that the low-frequency loss peak shifts more rapidly toward higher frequencies than the high-frequency one.

\subsection{Effects of Cooperative Interaction and Phase Transitions}

Cooperative interaction will in general tend to accelerate the convergence of the relaxation times with increasing temperature, because such interaction may be thought of as causing the barrier system to become more uniform [3, 5, 9]. For the models given in this paper this effect may be seen on a qualitative basis by letting $V$ tend to decrease with temperature. This leads to a more rapid increase of $g$ than if $V$ were invariant with temperature, and the net result is the aforementioned acceleration of the convergence of the relaxation times.

Cooperative interaction can lead to the existence of sharp phase transitions, where $V$ drops abruptly and $g$ increases from a small value to one near unity (fig. 5). This corresponds to the rapid equalizing of the barrier system at the transition temperature. With regard to the change of $V$ with temperature, examples close to the present case may be found in the literature [13].

It is of interest to discuss the changes to be expected in the loss curves, static dielectric constant,

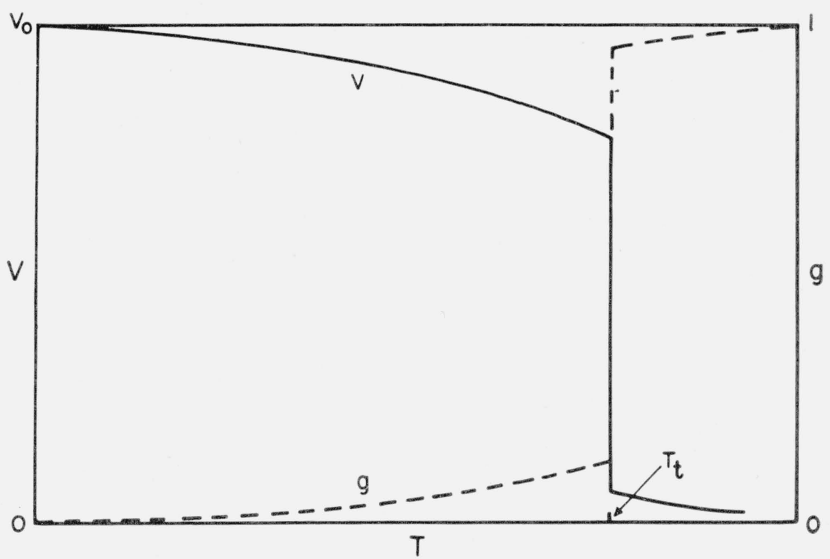

Figure 5. Behavior of $V$ (solid line) and $g$ dashed line as "a function of temperature in the vicinity of a sharp phase transition at $T_{t}$ (schematic).

crystal structure, and in the configurational entropy above and below such a sharp transition for models like $0-1$ and $0-2$.

Consider first the loss curves. It is clear from the sharp change in $g$ at the transition that an abrupt change may take place in the shape of the loss curves. This change will be especially marked if the phase just below the transition has a bimodal loss character, but may be much less so if the loss curve below the transition is quite narrow. The calculations also indicate that an abrupt decrease is to be expected in the "average" relaxation time as the temperature is raised through the transition, and this will cause the loss curve to shift toward higher frequencies.

In the simplest situation, the phase below the transition for models $0-1$ and $0-2$ will be $b c o$, and that above it $b c c$, the latter corresponding to the highly disordered lattice where the protuberance points at random in the five possible sites. As indicated previously, the bco phase could be bimodal (particularly at low temperatures) but it is unlikely that the isotropic $b c c$ phase would exhibit such behavior. For all the models discussed in this paper the configurational entropy at the absolute zero of temperature is zero, because each dipole has but one possible orientation. Above the transition where the phase is rotationally disordered, the configurational entropy assumes the value $R \ln 5$ entropy units. Hence the transition $b c o \rightarrow b c c$ would involve a maximum entropy of transition of $R \ln 5$ entropy units. ${ }^{6}$ The actual value will be slightly less than this due to the slight premonitory increase of disorder below the transition [note the slight increase of $g$ in figure 5 below the transition].

A more complicated set of phase transitions is possible for the $b c o$ lattice: the entropy might be gained in the steps $b c o \rightarrow b c t \rightarrow b c c$. In such a case the sum of the entropies of transition would still be $\sim R \ln 5$ entropy units. Furthermore, it is to be expected that, if bimodal loss behavior should occur, it is most likely to be found in the ordered bco phase (compare models $0-1,0-2, \mathrm{~T}-1$ ). Bimodal

${ }_{6}$ This refers to the entropy change for a constant volume process. Experimental entropies may contain a fairly large contribution due to the expansion of the crystal. 
loss curves might possibly appear for the bct phase if it is sufficiently ordered above the transition, but such behavior is not to be expected for the $b c c$ form.

The static dielectric constant tends to increase below the transition even if $V$ is held constant. In the cooperative case, it will tend to do so more rapidly, and will rise abruptly to a liquidlike value at the transition owing to the sharp increase of $g$ at that temperature. The above holds for the transition $b c o \rightarrow b c c$, where $V \cong 0, g \cong 1$ above the transition, but in the case of the more complicated sequence $b c o \rightarrow b c t \rightarrow b c c$, an abrupt rise in dielectric constant may take place at either transition depending on the relative stability of the deep site in the bct form.

The remarks in the above paragraphs pertaining to the behavior of the dielectric constant, configurational entropy, crystal structure, and shape of the loss curves in the vicinity of cooperative phase transitions apply to the corresponding face-centered systems provided the eight sites due to the triads of nearest-neighbors are sufficiently unstable so that they may be ignored in comparison with the five orthogonal sites where the central dipole may point toward next-nearest neighbors (see section 3.1).

\subsection{Qualitative Predictions and Comparison With Experiment}

It is considered that the work with three-dimensional models with one deep site is sufficiently complete and general to cite certain qualitative predictions concerning the nature of the bimodal loss curves which can arise in such systems. With certam qualifications which will be noted, the predictions mentioned below hold for all such models which have been studied (many unpublisbed). The basic reasoning leading to these predictions is mainly to be found in the preceding discussion. In general, the theory indicates that:

(a) Bimodal loss curves are most apt to be found at low temperatures in anisotropic phases (such as the orthorhombic) where the dielectric constant is relatively low and $d \epsilon_{s} d T$ is positive. They are unlikely to arise in highly disordered and isotropic systems, especially where the dielectric constant is relatively high and has the negative temperature coefficient typical of a polar liquid.

(b) The two loss peaks will tend to converge with increasing temperature, and the loss peak connected with the longer relaxation time will shift toward higher frequencies more rapidly than that associated with the shorter relaxation time.

(c) The relative magnitude of the two loss peaks will change with temperature, but in a few special cases this effect may be quite small (see model T-1)

These predictions may easily be extended to include the changes expected in the vicinity of solid-state phase transitions. If the low-temperature phase is bimodal just below the transition, it is commonly to be expected that only a single (though perhaps highly broadened) loss peak will appear in the more isotropic high-temperature form, and that the mean relaxation time of the latter will be as short or shorter than that obtained for either of the peaks below the transition. If the two peaks of the bimodal loss curve have already coalesced prior to the transition, a further narrowing may take place at the transition, but the most marked effect in this case will probably be an abrupt shift of the loss maximum to a higher frequency. It should be noted, however, that the persistence of the bimodal effect above a phase transition is not rigidly excluded by the theory: a possible example would be the $b c o \rightarrow b c t$ transition where the bct phase remained ordered.

Some aspects of the general validity of the theory may be examined by comparing the predictions given above with what has been found experimentally for the $f c o, f c t$, and $f c c$ structures in the hydrogen halides. It is believed that such a qualitative comparison is permissible, even though the calculations on which the predictions are based refer directly only to the $b c o, b c t$, and $b c c$ structures. ${ }^{7}$ First, it will be recalled that under certain circumstances (section 3.1) the results for the body-and face-centered lattices may be identical. Second, the predictions listed are just those that are insensitive to the particular three-dimensional single deep site model chosen, and we have been unable to uncover any reasonable models of the type mentioned above where these predictions failed generally. Finally, even if the hydrogen halides are hydrogen-bonded, so that the protuberance (hydrogen) points toward a nearest neighbor, thus forming sheets of zigzag chains [14] where the sites may be nonorthogonal, there seems to be no valid reason for abandoning the orthogonal orientational model with one deep site as a useful first approximation. In the hydrogen-bonded "site" model, the deep site would simply be the primary hydrogen-bonded position.

A summary of the dielectric investigations of Cole and coworkers $[9,10,11,12]$ on the solid hydrogen halides is given in table 3 . Insofar as the necessary data can be obtained from their work, the general qualitative predictions given above appear to hold. Note that bimodal behavior is found in the most anisotropic (orthorhombic) phases in accord with (a), and that the two loss peaks undergo changes with temperature of the type mentioned in (b) and (c).

As mentioned in section 4, bimodal behavior is not to be expected for a tetragonal lattice that is disordered to such an extent that the static dielectric constant behaves in a manner similar to that of a liquid (e. g., $\epsilon_{s}$ is large and $d \epsilon_{s} / d T$ is negative). The tetragonel forms mentioned in table 3 exhibit negative values of $d \epsilon_{s} / d T$, and insofar as the nature of the loss curves has been elucidated, only single loss peaks are found. It should be mentioned,

\footnotetext{
HCN possesses a $b c o$ structure similar in appearance to that depicted in figure 1 (a) except that the dipoles are all parallel to one another [see W. J. Dulmage and W. N. Lipscomb, Acta Cryst. 4, 330 (1951)]. Fortunately, the same model (fig. 1 (b)) holds for both the parallel and antiparallel cases. At higher temperatures, HCN has a bct structure. The parallel dipole bct structure leads to the site model shown in figure 4 , so that the theory given for model T-1 may well apply to this phase. Hence, a direct application of the theory to body-centered systems may be possible when sufficient dielectric data are available for such systems. An application should not be attempted if the parallel case leads to
} ferroelectric behavior, but this is not anticipated in the case cited. 
TABLE 3. Dielectric behavior of the hydrogen halides (after Cole and coworkers)

\begin{tabular}{|c|c|c|c|}
\hline Substance & Low-temperature $f c o$ form (phase III) & $\begin{array}{l}\text { Intermediate-temperature } f_{c t} \text { form (phase } \\
\text { II) }\end{array}$ & High-temperature $f c c$ form (phase I) \\
\hline $\mathrm{HCl}{ }^{9} \ldots$ & $\left\{\begin{array}{l}\text { Bimodal at low temperatures. The two loss } \\
\text { peaks converge with rising temperature with } \\
\text { a change in relative magnitude, coalescing } \\
\text { just prior to the first-order transition. The } \\
\text { low-frequency peak shifts toward higher fre- } \\
\text { quencies with rising temperature more rapidly } \\
\text { than the high-frequency one. } \epsilon_{s} \text { low, } d \epsilon_{s} / d T \\
\text { positive. }\end{array}\right.$ & $\begin{array}{l}\text { This phase absent in } \mathrm{HCl} \text {; } f c o \text { form con- } \\
\text { verts directly to } f c c \text { form at transition. }\end{array}$ & $\begin{array}{l}\text { Dielectric loss not investigated. Di- } \\
\text { electric constant similar to that of } \\
\text { a polar liquid }\left(\epsilon_{s} \text { large, } d \epsilon_{s} / d T \text { nega- }\right. \\
\text { tive). }\end{array}$ \\
\hline $\mathrm{HBr}{ }^{10,12}, \mathrm{DBr}^{11} \ldots$ & $\left\{\begin{array}{l}\text { Bimodal at low temperatures. Relative magni- } \\
\text { tude of the two loss peaks change with tem- } \\
\text { perature. May be bimodal up to transition. } \\
\text { Low-frequency peak shifts toward higher } \\
\text { frequencies more rapidly than higb-frequency } \\
\text { ones. } \epsilon_{s} \text { unusually high near } \lambda \text {-transition, but } \\
d \epsilon_{s} / d T \text { positive. }\end{array}\right.$ & $\begin{array}{l}\text { Debye-type on low-frequency side of loss } \\
\text { curve, but high-frequency data insuffici- } \\
\text { ent to fully determine shape. Loss max- } \\
\text { imum is at higher frequency than in fco } \\
\text { form. } \epsilon_{s} \text { unusually high near } \lambda \text {-transi- } \\
\text { tion, } d \epsilon_{s} / d T \text { negative. }\end{array}$ & $\begin{array}{l}\text { Dielectric loss not investigated. Di- } \\
\text { electric constant similar to that of a } \\
\text { polar liquid }\left(\epsilon_{s} \text { large, } d \epsilon_{s} / d T \text { nega- }\right. \\
\text { tive }) \text {. }\end{array}$ \\
\hline $\mathrm{HI}^{11}, \mathrm{DI}^{11} \ldots$ & $\left\{\begin{array}{l}\text { Loss curve highly broadened but not bimodal } \\
\text { in temperature range studied. } \epsilon_{s} \text { low, } d \epsilon_{s} / d T \\
\text { positive. }\end{array}\right.$ & $\begin{array}{l}\text { Debye-type over considerable frequency } \\
\text { range but shape of loss curve not fully } \\
\text { defined on high-frequency side. Loss } \\
\text { maximum is at higher frequency than } \\
\text { in } f c o \text { form. Dielectric constant liquid- } \\
\text { like ( } \epsilon_{s} \text { large, } d \epsilon_{s} / d T \text { negative). }\end{array}$ & $\begin{array}{l}\text { Dielectric loss not investigated. Di- } \\
\text { electric constant similar to that of } \\
\text { a polar liquid }\left(\epsilon_{s} \text { large, } d \epsilon_{s} / d T \text { nega- }\right. \\
\text { tive). }\end{array}$ \\
\hline
\end{tabular}

however, that any such qualitative comparison may well be invalid in the immediate vicinity of the lambda transition between the fco and fct phases in $\mathrm{HBr}$ and $\mathrm{DBr}$ where the dielectric constant reaches very high values. These high dielectric constant values indicate a strong dipolar correlation effect not taken into account in the polarizability calculations. Such an effect does not occur near the first-order transition in hydrogen chloride, and is small near the lambda transition in hydrogen iodide. The substitution of deuterıum for hydrogen sometimes leads to large increases in the observed relaxation times [11. Such a phenomenon can be understood qualitatively if it is assumed that quantum tunnelling affects some of the transition probabilities.

It is worth pointing out that the properties of $f_{c o}$ hydrogen chloride [9] are similar to those of model $0-2$. The loss curves are of approximately the type depicted in figure 3 , and the static dielectric constant rises with increasing temperature. Also, the entropy of the first-order transition has been listed by Powles [8] as $R \ln 4.7$ entropy units, which is consistent with that suggested for model $0-2$ (section 5.3).

The general type of theory proposed here and in earlier work may be checked further by examining the properties of substances that possess loss curves with only a single maximum. The theory indicates that the relaxation times will generally converge with increasing temperature, and this leads to the prediction that broadened loss curves confined to a single maximum should become narrower with increasing temperature. ${ }^{8}$ In the cases known to the authors where sufficient data are available to test it, this prediction is generally borne out by experiment; there appear to be no cases where a loss curve broadens with rising temperature.

\footnotetext{
${ }^{8}$ Results of dielectric investigations are frequently reported in the form of an Argand diagram (Cole and Cole semicircular arc plot). In terms of this presen. tation the prediction is that if the center of the circle describing the experimental points is depressed below the $\epsilon^{\prime}$ ordinate, it should rise toward it as the temperature is raised. Stated in another way, if the Cole and Cole parameter $\alpha$ is initially greater than zero, it should decrease with rising temperature.
}

\subsection{Bimodal Loss Curves Due to Other Causes}

It seems necessary to mention that bimodal loss curves in real materials can arise from a trivial cause, namely, that two distinct phases, each with its own relaxation time, may coexist during the measurement. Under equilibrium conditions such a twophase system will, of course, not occur except at melting or first-order transition temperatures, but it is well known that two phases (at least one being metastable) have a tendency to coexist over a wide range of temperatures in certain compounds. It is therefore clear that if bimodal loss curves are found experimentally, it should not be assumed that they are a property belonging to a single phase without conducting proper checks aimed at detecting a second phase. X-ray diffraction experiments can often distinguish between monophase and two-phase systems. Also, if at any given temperature the relative magnitudes of two loss peaks depend markedly on the thermal or mechanical history of the sample, it is quite likely that two or more phases may be present. There is no indication that any of the experimental bimodal loss curves mentioned in this paper are due to the presence of two phases.

Resonance absorption due to oscillatory motions of dipoles may occur at microwave or higher frequencies in polar solids. If a material shows absorption due to this effect, and further exhibits a typical dipole absorption at lower frequencies, a bimodal loss curve will result. The present theory exhypothesi deals only with dipole orientation, and does not account for bimodal loss curves where a high frequency peak is due to the resonance effect. Resonance absorption will lead to a loss peak that is narrower than that predicted by the Debye equation, and is restricted on theoretical grounds to frequencies corresponding to relaxation times which are $\sim 10^{-12}$ sec or shorter. The validity of a site model, as well as that of the $S-J$ hypothesis, depends on the presence of barriers which are in excess of the prevailing thermal energy $\boldsymbol{k} T$. To be on the safe side, it is 
best to assume that each of the $W_{i j}$ is at least $5 \boldsymbol{k} T$. With the expression $\tau=(h / \boldsymbol{k} T) \exp \left(W_{i j} / \boldsymbol{k} T\right)$ one then obtains the rough estimate that the present theory is valid for systems with relaxation times of $\sim 10^{-10}$ sec or longer. Another way of estimating the range of validity of the present theory is to observe that the shortest relaxation time which one might expect due to dipole rotation must certainly be somewhat longer than that calculated for free rotation [5]: this again leads to the suggestion that the theory should be valid for relaxation times which are $\sim 10^{-10}$ sec or longer. Thus, an incorrect application of the theory resulting from the intrusion of resonance absorption can generally be avoided simply by dealing with data obtained at frequencies not much in excess of $10^{9}$ or $10^{10}$ cycles per second. In many cases it should be possible to establish the absence of resonance absorption and thus treat data obtained at somewhat higher frequencies.

\subsection{Fluctuations}

It has been assumed in the present type of theory that, as the selected central dipole undergoes reorientations, the nearest neighbors remain fixed. This is considered to be an excellent approximation as long as the temperature is low and most of the molecules are in the ground state, and such a state of affairs should prevail for values of the parameter $g=\exp (-V / \boldsymbol{k} T)$ that range from zero to roughly 0.01 or 0.1 . However, when one of the nearest neighbors to the central molecule is itself out of position owing to thermal agitation, it is no longer strictly correct to main tain the same set of transition probabilities for processes involving jumps past this particular neighbor. The approximation implicitly used here and in earlier papers in dealing with this situation is equivalent to assuming that such fluctuations of structure can be "averaged" for the central dipole, which is reminiscent of the Bragg-Williams approximation commonly used in equilibrium orderdisorder [15] and orientational disorder [13] theory. The effect of fluctuations of this type would be most marked in disordered crystals at high temperatures; this condition corresponds to $g \cong 1$ where almost all of the molecules are in "wrong" orientational positions.

There are good reasons for believing that fluctuations of structure of the type described above lead to only second-order effects in the dielectric relaxation spectrum of monophase molecular crystals, and that it is therefore misleading to attribute to this cause alone the broadening of loss curves in such systems.

In the first place, it is clear that if fluctuations of structure played the predominant role in causing the broadening of loss curves, rising temperature would cause an increased broadening of the loss curves. As is brought out in section 5.4, this is contrary to all the presently available experimental evidence concerning the temperature-dependence of the shape of dielectric loss curves. Second, if it is assumed that fluctuations are the sole cause of the broadening of loss curves, it is very difficult to see how bimodal loss curves could arise in monophase crystals. A third point is that a few crystals exhibit a Debye-type loss curve (single relaxation time) at all temperatures. Such a phenomenon can be understood, at least to a first approximation, in terms of an isotropic crystalline field (or certain special site models), but adopting the fluctuation viewpoint, it would appear to be necessary to arbitrarily postulate the total absence of fluctuations at all temperatures to explain such a result.

Noting that the present theory leads in a natural way to the correct type of temperature-dependence of the shape of the loss curves, and that it can yield both bimodal and Debye-type curves, it is our conclusion that it is generally more satisfactory to attribute the existence of multiple dielectric relaxation times in monophase molecular crystals to the anisotropy of the crystalline field in which the dipoles reorient.

The authors thank F. Buckley, S. G. Weissberg, J. I. Lauritzen, and A. D. Franklin for making a number of helpful suggestions during the course of this work.

\section{References}

[1] J. D. Hoffman and B. M. Axilrod, J. Research NBS 54, 357 (1955) RP2598.

[2] B. M. Axilrod, J. Research NBS 56, 81 (1956) RP2651.

[3] J. D. Hoffman, J. Chem. Phys. 23, 1331 (1955).

[4] P. J. W. Debye, Polar molecules, p. 94 (Dover Publications, New York, N. Y., 1945).

[5] J. D. Hoffman and H. G. Pfeiffer, J. Chem. Phys. 22, 132 (1954).

[6] J. G. Powles, J. Chem. Phys. 21, 633 (1953).

[7] J. J. O'Dwyer and R. A. Sack, Australian J. Science Research 5, 647 (1952).

[8] J. G. Powles, Trans. Faraday Soc. 48, 430 (1952).

[9] R. W. Swenson and R. H. Cole, J. Chem. Phys. 22, 284 (1954).

[10] N. L. Brown and R. H. Cole, J. Chem. Phys. 21, 1920 (1953).

[11] S. Havriliak, Jr., and R. H. Cole, J. Chem. Phys. 23, 2455 (1955).

[12] N. L. Brown and R. H. Cole, J. Chem. Phys. 20, 196 (1952).

[13] J. D. Hoffman, J. Chem. Phys. 20, 541 (1952)

[14] D. F. Hornig and W. E. Osberg, J. Chem. Phys. 23, 662 (1955).

[15] J. Frenkel, Kinetic theory of liquids, chap. II (Oxford Univ. Press, London, 1949). R. F. Fowler and E. A. Guggenheim, Statistical thermodynamics (Cambridge Univ. Press, 1949).

Washington, August 15, 1956. 